\title{
Which size is the best cutoff for primary small gastric gastrointestinal stromal tumor?
}

\author{
Xingyu Feng ${ }^{1 \#, ~ Z i f e n g ~ Y a n g ~}{ }^{2 \#}$, Peng Zhang ${ }^{3}$, Tao Chen ${ }^{4}$, Haibo Qiu ${ }^{5}$, Zhiwei Zhou ${ }^{5}$, Guoxin Li $^{4}$, \\ Kaixiong Tao ${ }^{3}$, Hui Wang ${ }^{2}$, Yong $\mathrm{Li}^{1}$ \\ ${ }^{1}$ Department of General Surgery, Guangdong Provincial People's Hospital, Guangdong Academy of Medical Sciences, Guangzhou 510000, China; \\ ${ }^{2}$ Department of Colorectal Surgery, the Sixth Affiliated Hospital of Sun Yat-sen University, Guangdong Provincial Key Laboratory of Colorectal and \\ Pelvic Floor Diseases, the Sixth Affiliated Hospital of Sun Yat-sen University, Guangzhou 510000, China; ${ }^{3}$ Department of Gastrointestinal Surgery, \\ Union Hospital Tongji Medical College Huazhong University of Science and Technology, Wuhan 430000, China; ${ }^{4}$ Department of General Surgery, \\ Southern Medical University Nanfang Hospital, Guangzhou 510000, China; ${ }^{5}$ Department of Gastric and Pancreatic Surgery, Sun Yat-sen University \\ Cancer Center, State Key Laboratory of Oncology in South China, Collaborative Innovation Center of Cancer Medicine, Guangzhou 510000, China \\ Contributions: (I) Conception and design: X Feng, Z Yang; (II) Administrative support: None; (III) Provision of study materials or patients: Y Li; (IV) \\ Collection and assembly of data: P Zhang, T Chen; (V) Data analysis and interpretation: H Qiu; (VI) Manuscript writing: All authors; (VII) Final \\ approval of manuscript: All authors. \\ \#These authors contributed equally to this work as co-first authors. \\ Correspondence to: Yong Li, MD, PhD. Guangdong Provincial People's Hospital, Guangdong Academy of Medical Sciences, Guangzhou 510000, \\ China. Email: sywcwktg@126.com; Hui Wang, MD. Department of Colorectal Surgery, the Sixth Affiliated Hospital, Sun Yat-sen University, \\ Guangzhou, 510000 China. Email: wang89@mail.sysu.edu.cn.
}

Background: The biological behavior of primary small gastric gastrointestinal stromal tumor (gGIST) is indolent. The cutoff size categorizing small gGIST continues to be controversial. To date, there is no consensus regarding whether it should be $1 \mathrm{~cm}, 2 \mathrm{~cm}$, or another size. We aimed to find a new cutoff size.

Methods: Retrospective clinicopathological and prognosis data of patients with small gGIST from January 1998 to January 2015 were collected among five medical centers in southern China. Tumor size was divided into two groups: $<1 \mathrm{~cm}$ (Mirco group) and 1-2 cm (Small group). We compared the clinicopathological index and prognosis between these two groups and identified a new cutoff size to define small gGIST.

Results: During this 18-year period, there were 276 patients with primary small gGIST treated at these five medical centers. The range of tumor size was $0.2-2.0 \mathrm{~cm}$. The median tumor size was $1.0 \mathrm{~cm}$. The range of the mitotic count was $0-70 / 50$ high power fields (HPFs) with counts $\leq 5 / 50 \mathrm{HPFs}$ in 259 patients (93.8\%), $5<$ counts $\leq 10 / 50 \mathrm{HPFs}$ in 7 patients (2.5\%), and counts $>10 / 50 \mathrm{HPFs}$ in 10 patients $(3.6 \%)$. The median follow-up time was 38 months (3-156 months). The 5 -year overall survival rate was $98.7 \%$ in the entire group. Using Pearson correlation analysis, there was a positive correlation between the mitotic count and tumor size as a continuous variable $(\mathrm{r}=0.164, \mathrm{P}=0.006)$. There were 137 patients in the Micro group and 139 cases in the Small group. In the Micro group, mitotic counts were $\leq 5 / 50$ HPFs in 134 patients, $5<$ counts $\leq 10 / 50 \mathrm{HPF}$ in 0 patients, and counts $>10 / 50 \mathrm{HPFs}$ in 3 patients; mitotic counts in the Small group were counts $\leq 5 / 50$ HPFs in 125 patients, $5<$ counts $\leq 10 / 50$ HPFs in 7 patients, $>10 / 50$ HPFs in 7 patients. There was a statistically significant difference between these two groups $(\mathrm{P}=0.002)$; the Small group had more intermediate/high-risk cases. Using the receiver operating characteristic curve (ROC curve), we observed that $1.15 \mathrm{~cm}$ was the new cutoff size to separate low-risk cases and intermediate/high-risk cases (AUC $=0.707, \mathrm{P}=0.004$, sensitivity $=0.824,1$-specificity $=0.429$ ).

Conclusions: Primary small gGIST has a good prognosis; gGIST $<1 \mathrm{~cm}$ can be regarded as benign tumors that only requires endoscopic ultrasonography (EUS) follow-up. The proportion of potential intermediate/ high-risk disease is high for patients with 1-2 cm gGIST. These patients should be treated with caution and the tumors should be resected if necessary. These results indicate that $1.15 \mathrm{~cm}$ may be the new cutoff size to separate small gGIST from large gGIST, but further studies are needed for verification. 
Keywords: Primary gastric gastrointestinal stromal tumor (primary gGIST); <1 cm gGIST; 1-2 cm gGIST; cutoff

Submitted Dec 30, 2019. Accepted for publication Feb 26, 2020.

doi: 10.21037/jgo.2020.03.08

View this article at: http://dx.doi.org/10.21037/jgo.2020.03.08

\section{Introduction}

Gastrointestinal stromal tumors (GISTs) originate from mesenchymal tissues and have a malignant tendency. The incidence rate is $1-4.7 / 10$ million per population (1-3). These tumors can occur anywhere within the digestive tract, and the most common location is the stomach (approximately 60\%) (3). The risk of recurrence and metastasis, according to the modified National Institutes of Health (NIH) grading system, is determined by factors including the tumor site, tumor size, mitotic counts and tumor rupture as the evaluation index, which is then stratified into very low, low, intermediate and high risk groups (4). In recent years, with diagnostic developments and the improvement of people's health awareness, the detection rate of primary small gastric gastrointestinal stromal tumor (gGIST) is approximately $20-30 \%$, which has gradually attracted the attention of clinicians (5-7). Related guidelines suggest that endoscopic ultrasonography (EUS) follow-up can be performed if there are no high-risk EUS features (irregularities, ulceration, echogenic foci and heterogeneity) or if the patient has no obvious and continuous clinical symptoms $(4,8)$. However, the cutoff size defining small gGIST is still controversial. The most widely recommended cutoff is $2 \mathrm{~cm}$, following the National Comprehensive Cancer Network (NCCN) guidelines that defined gGIST $\leq 2 \mathrm{~cm}$ as very small GIST (which is always called small GIST in the clinic) $(2,9)$. Several scholars have suggested that a $1 \mathrm{~cm}$ cutoff size should be used for small GISTs. Therefore, resection is recommended for patients with $1-2 \mathrm{~cm}$ GISTs (10). In this study, we retrospectively analyzed the clinicopathological and prognosis data of 276 patents with small gGIST in five medical centers in southern China from 1998 to 2015. All of the patients were divided into either the Micro group or the Small group. We compared the clinical features of the two groups and mapped the receiver operating characteristic (ROC) curve to identify the new cutoff size to predict intermediate/high-risk disease in patients with small gGIST.

\section{Methods}

We received ethical approval (No. GDREC2016296H) for this case series from five medical centers in southern China (Guangdong Provincial People's Hospital; Guangdong Academy of Medical Sciences, Guangzhou, China; Union Hospital Tongji Medical College Huazhong University of Science and Technology, Wuhan, China; Southern Medical University Nanfang Hospital, Guangzhou, China; Sun Yatsen University Cancer Center; State Key Laboratory of Oncology in South China; Collaborative Innovation Center of Cancer Medicine, Guangzhou, China; Sixth Affiliated Hospital, Sun Yat-sen University, Guangzhou, China), and each institution provided their patients' consent for publication.

\section{Patient information}

Patients with primary small gGIST $(\leq 2 \mathrm{~cm})$ were treated in five medical centers in southern China between 1998 and 2015. The tumor size was measured from a sample taken after resection. The enrollment criteria were a confirmed pathological diagnosis of gGIST treated with tumor resection. Patients were excluded from the study when death occurred due to other diseases, combined with other malignant tumors or multiple GIST case.

\section{Observations and follow-up}

The patients were grouped by 3 -year intervals into six different groups, namely, 1998-2000, 2001-2003, 2004-2006, 2007-2009, 2010-2012 and 2013-2015. Age, gender, tumor site, tumor size, surgical and pathological outcomes [immunohistochemistry was performed on $3-\mu \mathrm{m}$ sections and the following antibodies: CD117, CD34, Dog-1. Histological type (spindle, epithelioid, mixed) and mitotic index were also detected by hematoxylin and eosin stain], adjuvant therapy, genetic test (DNA of the GIST tissues was isolated using a QIAmp DNA FFPE Tissue kit. Polymerase chain reaction (PCR) was used to amplify 


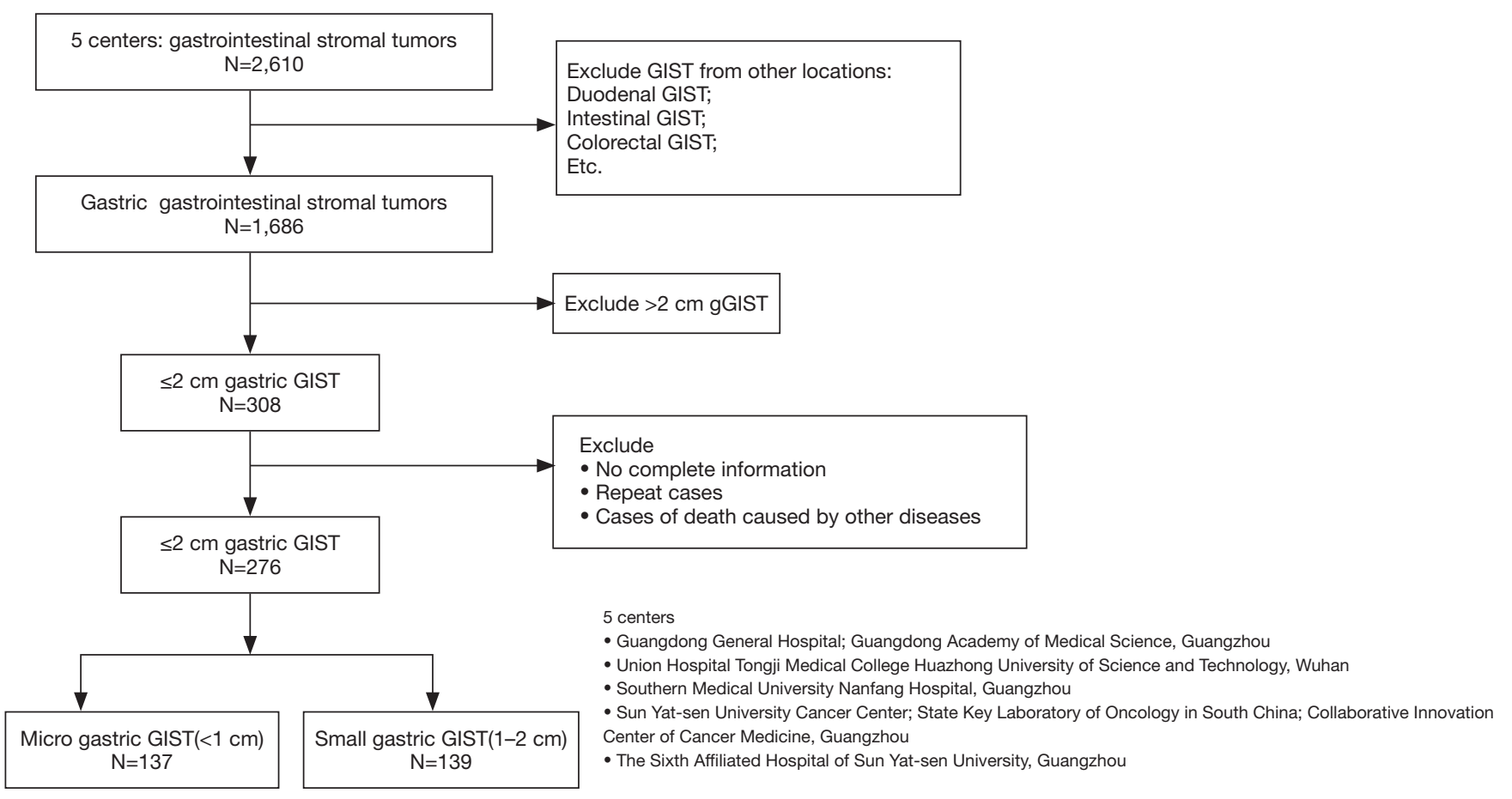

Figure 1 Study flowchart. GIST, gastrointestinal stromal tumor.

C-KIT exons 9, 11, 13 and 17 and PDGFRA exons 12 and 18. The PCR reaction was performed using a Taq PCR Master Mix) and other clinical and pathological factors were retrospectively analyzed. The tumor site was classified based on the anatomical location: gastric cardia, fundus, body, or antrum. Primary small gGIST was defined as $\leq 2 \mathrm{~cm}$ according to the NCCN guidelines; however, in this study, it was additionally subdivided into $<1 \mathrm{~cm}$ (Micro group) or $1-2 \mathrm{~cm}$ (Small group). Based on the modified NIH risk system, the patients were classified into the very low-, low, intermediate- or high-risk group. The mitotic count was assessed per 50 high power fields (HPFs). Pathological examinations were approved by three experienced pathologists from each institution. The last follow-up was February 15, 2016, or at the moment of tumor-related death.

\section{Statistical analysis}

Statistical analyses were performed using SPSS software (version 22.0 for Windows; SPSS, Chicago, IL, USA). Categorical data were compared by the chi-square test or Fisher's exact test. Survival curves were derived from the Kaplan-Meier estimates, and the curves were compared using the log-rank test. The relationship between the mitotic count and tumor size was analyzed using the Pearson chi-square test. The optimal cutoff values for tumor size were analyzed using the ROC curve with the criterion variable "tumor size" and "intermediate/high-risk" as a conditional variable. $\mathrm{P}$ values $<0.05$ were considered statistically significant.

\section{Results}

\section{Patient information}

A total of 276 patients with primary small gGIST were enrolled in this study (Figure 1). The entire cohort comprised $130(47.1 \%)$ males and 146 females (52.9\%) patients aged between 27 and 91 years at the time of diagnosis. The median age was 60 years, and there were 139 (50.4\%) patients older than 60 . Based on the six categorical time periods, there were 2 patients with gGIST diagnosed in 1998-2000, 2 in 20012003, 13 in 2004-2006, 69 in 2007-2009, 85 in 2010-2012, and 105 in 2013-2015 (Figure 2).

\section{Comparison between groups}

The patient characteristics, surgical outcomes and 


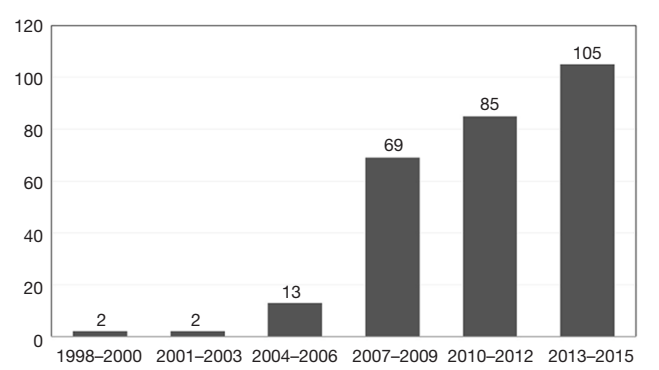

Figure 2 Cases of primary small gastric GIST in 6 different time periods. This shows an increasing tendency to perform resection for small gastric GISTs. GIST, gastrointestinal stromal tumor.

pathological data from the Micro and Small groups were well balanced (Tables 1, 2 and 3, respectively). In the whole group, the mitotic counts were $\leq 5 / 50,[5-10] / 50$, and $>10 / 50 \mathrm{HPFs}$ in 259 (93.8\%), 7 (2.5\%), and 10 (3.6\%) cases, respectively. Based on the histopathological classification, $268(97.1 \%)$ cases were classified as spindle cell type, $7(2.5 \%)$ as epithelial cell type and $1(0.4 \%)$ as a mixed type. Immunohistochemistry demonstrated that $74.6 \%$ (206/276), 98.2\% (271/276), and 97.4\% (269/276) of the patients had co-expression of CD34(+), CD117(+), and Dog-1(+), respectively. We found that there was a significant difference between the two groups in terms of mitotic count $(\mathrm{P}=0.002)$. Furthermore, based on the modified NIH risk criteria, there were 7 patients categorized as intermediate risk and 7 categorized as high risk in the Small group, while there were only 3 highrisk patients in the Micro group. A significant difference between the two groups in terms of the NIH risk system $(\mathrm{P}=0.002)$ (Table 3) was observed.

\section{Correlation between tumor size and mitotic count, and the ROC curve analysis for cut-off determination}

By making use of the Pearson correlation analysis, we found a positive correlation between the continuous variable mitotic count and tumor size $(\mathrm{r}=0.164, \mathrm{P}=0.006)$. A good linear relationship was observed during the comparison between the mitotic count and tumor size (Figure 3A). ROC curves were generated to find the sensitivity and specificity for detecting the optimal cutoff value to predict intermediate/high-risk disease (according to NIH criteria). The area under the curve (AUC) was $0.716(\mathrm{P}=0.003)$, indicating that the best cutoff value for tumor size to differentiate between the Micro and Small groups was $1.15 \mathrm{~cm}$. The AUC, sensitivity, specificity, positive predictive value, negative predictive value, and consistency rates were $0.716,82.4 \%, 59.5 \%, 12 \%, 98 \%$ and $60.9 \%$, respectively (Figure $3 B$ and Table 4).

\section{Survival}

Of the 276 patients with primary small gGIST, there were 220 patients with complete follow-up data. The median follow-up time was 38 months (3-156 months). Two $\mathrm{R} 1$ resection cases did additional surgery, the remaining one received imatinib treatment, all of them still be alive, no recurrence. There were two cases of recurrence (currently alive and receiving imatinib treatment), and an additional two high-risk patients died. The overall survival rate at 5 years was $98.7 \%$. There was no difference in survival between the Micro group and the Small group (Figure 4). Due to the lack of tumor progression and death data, we could not perform Kaplan-Meier univariate and Cox multivariate survival analyses.

\section{Discussion}

GISTs are the most common mesenchymal tumor of the digestive tract, and they are preferentially located in the stomach; the parameters of immunohistochemical expression are CD34, CD117 and Dog 1 (11). According to the modified NIH classification criteria, tumor site, tumor size, mitotic count and rupture are prognostic indicators. In recent years, with diagnostic developments and increased awareness of health improvements, as well as asymptomatic, autopsy and accidental cases (observed during surgery), increased the number of small GISTs diagnosed and created a detection rate of $3 \%$ to $35 \%(12,13)$. This study investigated 3 -year blocks as a sample time period. The results observed indicated that the incidence of small gGIST had a rapid increase in the 6 different time periods. At present, GISTs are divided into small size tumors (micro GISTs or small GISTs) and clinically significant tumors (overt-GIST, big GIST or large GIST). How to define a small GIST is still controversial, and 1 or $2 \mathrm{~cm}$ is a common cutoff value size. One study suggested that $3 \mathrm{~cm}$ might be a better cutoff (12). The current study refers to the most widely used NCCN guidelines; we defined primary gastric GIST $(\leq 2 \mathrm{~cm})$ as a small gGIST (4).

Small gGIST generally have self-limiting growth, and their biological behavior is indolent. The prognosis is good $(7,14)$. The overall 5 -year survival rate in this study was 
Table 1 Clinical information of two groups

\begin{tabular}{|c|c|c|c|c|c|}
\hline Clinical information & Subgroup & No. of cases (\%) & $<1 \mathrm{~cm}(\mathrm{~N}=137)$ & $1-2 \mathrm{~cm}(\mathrm{~N}=139)$ & $P$ value \\
\hline Age, years & $\leq 60$ & $137(49.6)$ & 66 & 71 & \\
\hline \multirow[t]{2}{*}{ Gender } & Male & $130(47.1)$ & 65 & 65 & 0.547 \\
\hline & Female & $146(52.9)$ & 72 & 74 & \\
\hline Presenting symptom & No & $64(23.2)$ & 34 & 30 & \\
\hline \multirow[t]{3}{*}{ Diagnostic workup } & CT & $231(83.7)$ & 119 & 112 & 0.225 \\
\hline & UGIE & $197(71.4)$ & 101 & 96 & \\
\hline & EUS & $182(65.9)$ & 88 & 94 & \\
\hline \multirow{3}{*}{ Tumor location } & Fundus & $107(38.8)$ & 54 & 53 & \\
\hline & Body & $117(42.4)$ & 57 & 60 & \\
\hline & Antrum & $28(10.1)$ & 13 & 15 & \\
\hline \multirow[t]{2}{*}{ Biopsy } & Yes & $11(4.0)$ & 5 & 6 & 0.724 \\
\hline & No & $265(96.0)$ & 132 & 133 & \\
\hline \multirow{2}{*}{$\begin{array}{l}\text { Postoperative adjuvant } \\
\text { therapy }\end{array}$} & Yes & $5(1.8)$ & 2 & 3 & 0.275 \\
\hline & No & $271(98.2)$ & 135 & 136 & \\
\hline Recurrence & Yes & $2(0.7)$ & 0 & 2 & 0.164 \\
\hline
\end{tabular}

CT, computed tomography; UGIE, upper gastrointestinal endoscopy; EUS, endoscopic ultrasound.

98.7\%. The NCCN developed treatment guidelines for very small gGIST $(\leq 2 \mathrm{~cm})$ in 2010 . The guidelines suggest that only EUS follow-up is required if there are no possible high-risk EUS features, including an irregular border, cystic spaces, ulceration, echogenic foci, and heterogeneity (4). However, we often encounter the following problems in clinical practice: EUS in the observation and diagnosis of small GISTs is worse than that for large GISTs; the high-risk EUS features need adequate clinical experience to determine the true prognosis, which means that lack of experience may lead to misdiagnosis (15). Otherwise, the related guidelines do not recommend biopsy for small gGIST; therefore, there is no special examination conducted to obtain the high mitotic counts and gene mutation of small gGIST to evaluate its risk grade $(3,16)$. In clinical practice, we can therefore see progress in size and observe malignant cases (intermediate/high-risk cases) after resection, as it is in the small gGIST follow-up process. Yang et al.'s study (17) showed that, in a total of 63 resected small gGIST cases $(\leq 2 \mathrm{~cm}), 14$ patients were intermediate/ high-risk cases (22.2\%), meanwhile, Akahoshi et al. (18) and Wang et al. (19) showed 23\% and 6.7\% were intermediate/ high-risk cases. In Gao et al.'s study (15), 16 (23.2\%) patients showed significant changes in tumor size during the followup of 69 minimal EUS-conducted GISTs. Hu et al. (20) found that most $1-3 \mathrm{~cm}$ gastric submucosal tumors $(71.6 \%)$ were indolent. Tumor progression was found only in 25 GIST patients (28.4\%). In our study, there were 17 intermediate/high-risk patients (6.2\%). Thus, screening out patients with intermediate/high-risk cases of small gGIST is still a clinical challenge $(10,21)$.

Tumor size and mitotic count are two important factors that affect the prognosis of small gGIST (22). Studies have shown that gGIST $<1 \mathrm{~cm}$ are considered to be at low risk of 
Table 2 Surgical outcome of two groups

\begin{tabular}{|c|c|c|c|c|c|}
\hline Surgical outcome & Subgroup & No. of cases (\%) & $<1 \mathrm{~cm}(\mathrm{~N}=137)$ & $1-2 \mathrm{~cm}(\mathrm{~N}=139)$ & $P$ value \\
\hline \multirow[t]{2}{*}{ Surgical method } & Laparoscopy & $137(49.6)$ & 65 & 72 & 0.622 \\
\hline & Laparotomy & $75(27.2)$ & 38 & 37 & \\
\hline \multirow[t]{4}{*}{ Resection scope } & $\mathrm{ER}$ & $64(23.2)$ & 34 & 30 & 0.159 \\
\hline & WR & $172(62.3)$ & 87 & 85 & \\
\hline & $D G$ & $19(6.9)$ & 7 & 12 & \\
\hline & TG & $14(5.1)$ & 6 & 8 & \\
\hline \multirow[t]{3}{*}{ Complications } & Yes & $8(2.9)$ & 4 & 4 & 1.000 \\
\hline & Bleeding & 2 & 1 & 1 & \\
\hline & No & $268(97.1)$ & 133 & 135 & \\
\hline
\end{tabular}

ER, endoscopic resection; WR, wedge resection; PG, proximal gastrectomy; TG, total gastrectomy.

Table 3 Pathological outcome of two groups

\begin{tabular}{|c|c|c|c|c|c|}
\hline Pathological outcome & Subgroup & No. of cases (\%) & $<1 \mathrm{~cm}(\mathrm{~N}=137)$ & $1-2 \mathrm{~cm}(\mathrm{~N}=139)$ & $P$ value \\
\hline \multirow[t]{2}{*}{ Mitotic count } & $\leq 5 / 50$ HPFs & $259(93.8)$ & 134 & 125 & 0.002 \\
\hline & $>5 / 50$ HPFs, $\leq 10 / 50$ HPFs & $7(2.5)$ & 0 & 7 & \\
\hline \multirow{2}{*}{$\begin{array}{l}\text { Histopathological } \\
\text { classification }\end{array}$} & Spindle & $268(97.1)$ & 134 & 134 & 0.675 \\
\hline & Epithelioid & $7(2.5)$ & 3 & 4 & \\
\hline \multirow[t]{3}{*}{$\mathrm{IHC}$} & CD34(+) & $206(74.6)$ & 101 & 105 & 0.346 \\
\hline & CD117(+) & $271(98.2)$ & 134 & 137 & \\
\hline & Dog-1(+) & $269(97.4)$ & 133 & 136 & \\
\hline \multirow[t]{2}{*}{ Tumor necrosis } & Yes & $4(1.5)$ & 1 & 3 & 0.370 \\
\hline & No & $272(98.6)$ & 136 & 136 & \\
\hline \multirow[t]{3}{*}{ NIH criteria } & Very low & $259(93.8)$ & 134 & 125 & 0.002 \\
\hline & Intermediate & $7(2.5)$ & 0 & 7 & \\
\hline & High & $10(3.6)$ & 3 & 7 & \\
\hline \multirow[t]{6}{*}{ Genetic test } & Yes & $39(14.1)$ & 19 & 20 & 0.347 \\
\hline & C-KIT 9 & 3 & 1 & 2 & \\
\hline & C-KIT 11 & 31 & 16 & 15 & \\
\hline & PDGFRA 18 & 2 & 0 & 2 & \\
\hline & No-mutation & 3 & 2 & 1 & \\
\hline & No & 237 (85.9) & 118 & 119 & \\
\hline
\end{tabular}

HPF, high power field; IHC, immunohistochemistry; NIH, National Institute of Health. 

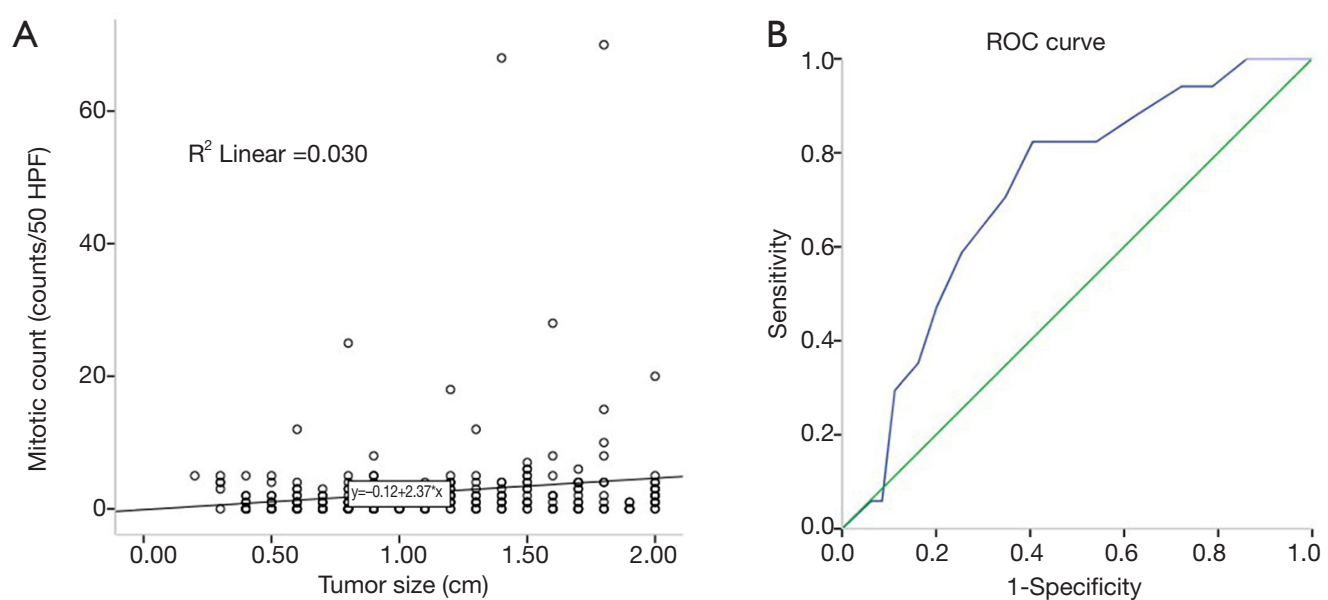

Figure 3 Correlation test and ROC curve test. (A) A linear relationship was observed when comparing mitotic count and tumor size using the Pearson chi-square test $(\mathrm{r}=0.164, \mathrm{P}=0.006)$; (B) receiver operating characteristic curve. The cutoff size for defining the small gastric GIST is $1.15 \mathrm{~cm}$. GIST, gastrointestinal stromal tumor; ROC, receiver operating characteristic.

Table 4 Coordinates of the curve [test result variable(s): tumor size]

\begin{tabular}{|c|c|c|}
\hline Positive if greater than or equal to ${ }^{a}$ & Sensitivity & 1-Specificity \\
\hline-0.8000 & 1.000 & 1.000 \\
\hline 0.2500 & 1.000 & 0.996 \\
\hline 0.3500 & 1.000 & 0.977 \\
\hline 0.4500 & 1.000 & 0.915 \\
\hline 0.5500 & 1.000 & 0.861 \\
\hline 0.6500 & 0.941 & 0.788 \\
\hline 0.7500 & 0.941 & 0.722 \\
\hline 0.8500 & 0.882 & 0.629 \\
\hline 0.9500 & 0.824 & 0.541 \\
\hline 1.0500 & 0.824 & 0.471 \\
\hline 1.1500 & 0.824 & 0.405 \\
\hline 1.2500 & 0.706 & 0.347 \\
\hline 1.3500 & 0.647 & 0.301 \\
\hline 1.4500 & 0.588 & 0.255 \\
\hline 1.5500 & 0.471 & 0.201 \\
\hline 1.6500 & 0.353 & 0.162 \\
\hline 1.7500 & 0.294 & 0.112 \\
\hline 1.8500 & 0.059 & 0.085 \\
\hline 1.9500 & 0.059 & 0.062 \\
\hline 3.0000 & 0.000 & 0.000 \\
\hline
\end{tabular}

The test result variable(s): tumor size has at least one tie between the positive actual state group and the negative actual state group. ${ }^{\text {a }}$, the smallest cutoff value is the minimum observed test value minus 1 , and the largest cutoff value is the maximum observed test value plus 1. All the other cutoff values are the averages of two consecutive ordered observed test values. malignancy; recommendations are to treat these tumors as benign tumors with regular EUS at follow-up (20). In this study, 276 patients with small gGIST were divided into two groups, the $<1 \mathrm{~cm}$ gGIST (Micro group) and the 1-2 cm gGIST (Small group). There was no difference in clinical, surgical or pathological information between the two groups. In the mitotic count and NIH criteria, we observed a higher mitotic count $>5 / 50$ HPFs and a concentration of high-risk patients in the Small group; there were statistically significant differences in the two groups $(\mathrm{P}=0.002)$ (Table 3). In Yang et al.'s study (17), there were 14 patients with intermediate/high-risk disease from 63 resected cases of small gGIST, of which 10 cases occurred in the Small group (1-2 cm), and it was suggested that gGIST of $1-2 \mathrm{~cm}$ should be excised. In our study, because R0 resection was performed in almost all cases, the Small group and the Micro group did not differ in survival. We also analyzed the correlation between the mitotic count and tumor size, and the results showed that the mitotic count showed an increasing trend with increasing tumor size, and there was a positive correlation $(\mathrm{R}=0.164, \mathrm{P}=0.006)$; this result was consistent with Fang et al. (23). Therefore, in our study, we consider the widely accepted " $2 \mathrm{~cm}$ criteria" to not be the best cutoff size to separate the low- and intermediate/ high-risk cases. Several past studies have shown that, for EUS that considers small gGIST, the best cutoff size was that associated with tumor progression, and the results were $9.5 \mathrm{~mm}(15), 14 \mathrm{~mm}(20,23)$ and $17 \mathrm{~mm}$ (24). In our study, the ROC curve analysis identified $1.15 \mathrm{~cm}$ as the best predictor of intermediate/high-risk disease with good 


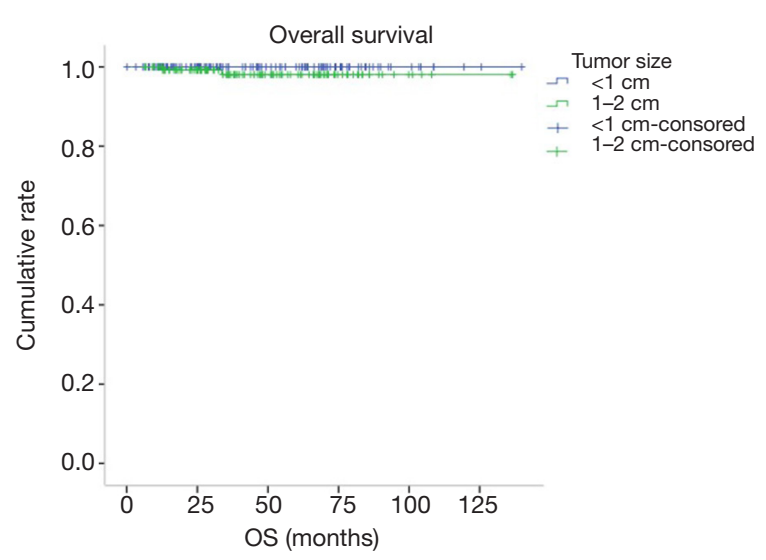

Figure 4 No difference in survival between the two groups $(\mathrm{P}=0.789)$.

sensitivity (82.4\%); the positive predictive value and negative predictive values were $12 \%$ and $98 \%$, respectively. We report that for the $\geq 1.15 \mathrm{~cm}$ gGIST, surgical intervention should be performed as soon as possible to confirm NIH risk and avoid progression.

There are several limitations of this study. First, this was a retrospective study. Second, because we lacked preoperative EUS results, it was difficult to analyze the reasons related to the choice of surgical resection. Third, the clinicopathological information of patients with intermediate/high-risk disease was incomplete; therefore, we cannot describe the characteristics of the patients with intermediate/high-risk disease. Multicenter randomized controlled studies should be conducted to confirm the benefit of surgical resection for small gastric GISTs compared with conservative treatment.

\section{Conclusions}

Detecting high-risk cases of small gGIST $(\leq 2 \mathrm{~cm})$ remains a clinical challenge. This study focused on the two prognostic indicators of tumor size and mitotic count. The results showed that more patients with high mitotic count (counts $>5 / 50 \mathrm{HPFs}$ ) and intermediate/high-risk disease were concentrated in the Small group $(1-2 \mathrm{~cm})$. At the same time, our study shows that $1.15 \mathrm{~cm}$ may be the new cutoff value to identify patients with intermediate/highrisk disease. Thus, patents with $1-2 \mathrm{~cm}$ gGIST tumors should receive active resection intervention, but as the micro gGIST $(<1 \mathrm{~cm})$ still bear malignant potential and the close observation is therefore necessary if lesions are not removed.

\section{Acknowledgments}

Funding: This study was supported by the Natural Science Foundation of Guangdong Province (No. 2016A030310328), Wu JiePing Medical Foundation (No. 201909) and GuangDong Science and Technology Cooperation Innovation and Platform Environmental Construction Fund (No. 2019A050510025).

\section{Footnote}

Conflicts of Interest: The authors have no conflicts of interest to declare.

Ethical Statement: The authors are accountable for all aspects of the work in ensuring that questions related to the accuracy or integrity of any part of the work are appropriately investigated and resolved. This case series were approved by five medical centers in southern China: Guangdong Provincial People's Hospital; Guangdong Academy of Medical Sciences, Guangzhou, China; Union Hospital Tongji Medical College Huazhong University of Science and Technology, Wuhan, China; Southern Medical University Nanfang Hospital, Guangzhou, China; Sun Yat-sen University Cancer Center; State Key Laboratory of Oncology in South China; Collaborative Innovation Center of Cancer Medicine, Guangzhou, China; Sixth Affiliated Hospital, Sun Yat-sen University, Guangzhou, China, and each institution provided their patients' consent for publication. All study procedures involving human participants were performed in accordance with the ethical standards of the institutional and/or national research committee and with the 1964 Helsinki Declaration and its later amendments or comparable ethical standards.

Open Access Statement: This is an Open Access article distributed in accordance with the Creative Commons Attribution-NonCommercial-NoDerivs 4.0 International License (CC BY-NC-ND 4.0), which permits the noncommercial replication and distribution of the article with the strict proviso that no changes or edits are made and the original work is properly cited (including links to both the formal publication through the relevant DOI and the license). See: https://creativecommons.org/licenses/by-nc-nd/4.0/.

\section{References}

1. Yang Z, Zheng R, Zhang S, et al. Incidence, distribution 
of histological subtypes and primary sites of soft tissue sarcoma in China. Cancer Biol Med 2019;16:565-74.

2. Coe TM, Fero KE, Fanta PT, et al. PopulationBased Epidemiology and Mortality of Small Malignant Gastrointestinal Stromal Tumors in the USA. J Gastrointest Surg 2016;20:1132-40.

3. Nishida T, Goto O, Raut CP, et al. Diagnostic and treatment strategy for small gastrointestinal stromal tumors. Cancer 2016;122:3110-8.

4. von Mehren M, Randall RL, Benjamin RS, et al. Soft Tissue Sarcoma, Version 2.2018, NCCN Clinical Practice Guidelines in Oncology. J Natl Compr Canc Netw 2018;16:536-63.

5. Mikami T, Nemoto Y, Numata Y, et al. Small gastrointestinal stromal tumor in the stomach: identification of precursor for clinical gastrointestinal stromal tumor using c-kit and alpha-smooth muscle actin expression. Hum Pathol 2013;44:2628-35.

6. Fujita T. Management of small gastrointestinal stromal tumours. Lancet 2013;382:1701.

7. Ma GL, Murphy JD, Martinez ME, et al. Epidemiology of gastrointestinal stromal tumors in the era of histology codes: results of a population-based study. Cancer Epidemiol Biomarkers Prev 2015;24:298-302.

8. Casali PG, Jost L, Reichardt P, et al. Gastrointestinal stromal tumors: ESMO clinical recommendations for diagnosis, treatment and follow-up. Ann Oncol 2008;19 Suppl 2:ii35-8.

9. Yamamoto K, Tsujinaka T, Takahashi T, et al. Impact of the Japanese gastric cancer screening system on treatment outcomes in gastric gastrointestinal stromal tumor (GIST): an analysis based on the GIST registry. Ann Surg Oncol 2015;22:232-9.

10. Soreide K. Cancer biology of small gastrointestinal stromal tumors $(<2 \mathrm{~cm})$ : What is the risk of malignancy? Eur J Surg Oncol 2017;43:1344-9.

11. Soreide K, Sandvik OM, Soreide JA, et al. Global epidemiology of gastrointestinal stromal tumours (GIST): A systematic review of population-based cohort studies. Cancer Epidemiol 2016;40:39-46.

12. Abraham SC, Krasinskas AM, Hofstetter WL, et al. "Seedling" mesenchymal tumors (gastrointestinal stromal tumors and leiomyomas) are common incidental tumors of the esophagogastric junction. Am J Surg Pathol 2007;31:1629-35.

13. Muenst S, Thies S, Went P, et al. Frequency, phenotype, and genotype of minute gastrointestinal stromal tumors in the stomach: an autopsy study. Hum Pathol
2011;42:1849-54.

14. Giuliano K, Nagarajan N, Canner J, et al. Gastric and small intestine gastrointestinal stromal tumors: Do outcomes differ? J Surg Oncol 2017;115:351-7.

15. Gao Z, Wang C, Xue Q, et al. The cut-off value of tumor size and appropriate timing of follow-up for management of minimal EUS-suspected gastric gastrointestinal stromal tumors. BMC Gastroenterol 2017;17:8.

16. Huang Z, Li Y, Zhao H, et al. Prognositic factors and clinicopathologic characteristics of small gastrointestinal stromal tumor of the stomach: a retrospective analysis of 31 cases in one center. Cancer Biol Med 2013;10:165-8.

17. Yang J, Feng F, Li M, et al. Surgical resection should be taken into consideration for the treatment of small gastric gastrointestinal stromal tumors. World J Surg Oncol 2013;11:273.

18. Akahoshi K, Oya M, Koga T, et al. Clinical usefulness of endoscopic ultrasound-guided fine needle aspiration for gastric subepithelial lesions smaller than $2 \mathrm{~cm}$. J Gastrointestin Liver Dis 2014;23:405-12.

19. Wang M, Xue A, Yuan W, et al. Clinicopathological Features and Prognosis of Small Gastric Gastrointestinal Stromal Tumors (GISTs). J Gastrointest Surg 2019;23:2136-43.

20. $\mathrm{Hu} \mathrm{ML}, \mathrm{Wu} \mathrm{KL}$, Changchien CS, et al. Endosonographic surveillance of 1-3 cm gastric submucosal tumors originating from muscularis propria. World J Gastroenterol 2017;23:2194-200.

21. Kanda T. Is a "wait-and-see" policy the best for small gastric gastrointestinal stromal tumor (GIST)? 'Transl Gastroenterol Hepatol 2016;1:1.

22. Navarrete A, Momblan D, Almenara R, Lacy A. Giant Gastric Gastrointestinal Stromal Tumor (GIST). J Gastrointest Surg 2017;21:202-4.

23. Fang YJ, Cheng TY, Sun MS, et al. Suggested cutoff tumor size for management of small EUS-suspected gastric gastrointestinal stromal tumors. J Formos Med Assoc 2012;111:88-93.

24. Lachter J, Bishara N, Rahimi E, et al. EUS clarifies the natural history and ideal management of GISTs. Hepatogastroenterology 2008;55:1653-6.

Cite this article as: Feng $\mathrm{X}$, Yang Z, Zhang $\mathrm{P}$, Chen T, Qiu H, Zhou Z, Li G, Tao K, Wang H, Li Y. Which size is the best cutoff for primary small gastric gastrointestinal stromal tumor? J Gastrointest Oncol 2020;11(2):402-410. doi: 10.21037/ jgo.2020.03.08 